\title{
EL LIDERAZGO FEMENINO Y SU EJERCICIO \\ EN LAS ORGANIZACIONES EDUCATIVAS
}

\author{
Enrique Javier Díez Gutiérrez y Rosa Eva Valle Flórez \\ Universidad de León, España. \\ Eloína Terrón Bañuelos \\ Trabajadora Social S.O.E.P., España. \\ Begoña Centeno Suárez \\ Orientadora I.E.S., España.
}

\begin{abstract}
"La mirada de todos esos hombres nada tiene que ver con la mía. No es ni mejor ni peor. Tan sólo diferente. Pero yo me veo obligada, cada día, a adaptar mis ojos a los suyos... Conservar nuestra propia mirada conociendo la suya, nos aporta sabiduría, independencia y libertad." (Ana Carrascosa, 2002, 13).
\end{abstract}

\section{INTRODUCCIÓN}

Toda la historia humana desde sus inicios está marcada profundamente por el patriarcado. Todas las culturas, todas las civilizaciones, todas las organizaciones lo han sufrido de una forma o de otra. Lo cual implica que a lo largo de la historia de la humanidad se ha generado una estructura social y cultural asentada en el predominio permanente de una parte de la población sobre otra por razón de sexo. Esto ha supuesto la consolidación de una concepción de las personas y de su función en la sociedad en función de su género. Es decir, se ha provocado una división del trabajo, una separación de los espacios, unas determinadas expectativas sobre habilidades, capacidades, destrezas, etc., de acuerdo con el género al que se pertenecía. $Y$ estos estereotipos tan arraigados son muy difíciles de erradicar en las prácticas cotidianas y en nuestra visión del mundo y de la realidad que nos rodea. La situación actual muestra una perpetuación de imágenes distorsionadas de la mujer que siguen marcando roles establecidos que priorizan la responsabilidad de la mujer en el ámbito doméstico frente al profesional.

En toda organización social es necesaria una coordinación de los diversos componentes que en ella participan. Aunar esfuerzos, consensuar estrategias para alcanzar las finalidades pretendidas, articular funciones, etc., son elementos imprescindibles en toda organización. Surge así el papel o la función de coordinación, aspecto esencial en las instituciones.

Hasta aquí podríamos estar todos y todas de acuerdo. Es necesaria la coordinación de los esfuerzos individuales en una organización para que converjan en un esfuerzo conjunto orientado en función de unas metas. Pero el problema surge cuando nos planteamos quién o quiénes tienen que asumir ese papel de coordinación.

El acceso de la mujer a puestos de dirección es una de las condiciones esenciales de la práctica de la igualdad en el seno de las organizaciones. Si esto no es habitual en las organizaciones educativas, el modelo de educación que se transmitirá contribuirá a desarrollar una educación no acorde con los planteamientos de la igualdad de oportunidades entre mujeres y hombres. $\mathrm{Y}$ esto es importante porque lo que las futuras generaciones de hombres y mujeres aprenden en la escuela, no es sólo lo que les transmitimos, sino también lo que ven y viven en la organización y funcionamiento de esas instituciones en las que pasan al menos 14 años de su vida. 
La educación es una profesión en donde predomina el número de mujeres. ¿Por qué, sin embargo, las estadísticas son tan persistentes en demostrar que el número de mujeres en puestos directivos en centros educativos es mucho menor de lo que correspondería en proporción a su presencia en esta profesión?

El acceso de las mujeres a puestos de dirección en las organizaciones educativas es un tema que ha surgido recientemente, por lo que las investigaciones y la bibliografía y documentación sobre el mismo (principalmente en español) son escasas y recientes. Por ello nos podemos cuestionar si se puede hablar de un modelo de liderazgo femenino, es decir, si las mujeres tienen un peculiar estilo de desempeñar el poder, de ejercer influencia en las organizaciones.

\section{EL LIDERAZGO Y LA DIRECCIÓN DE LAS MUJERES}

Como hemos dicho, hablar de liderazgo femenino es muy ambiguo. De hecho toda categorización que conlleva atribuir una "cualidades" o "rasgos" a un colectivo, no sólo supone un enfoque psicologicista e individualista, que ya cuestionamos anteriormente, sino que asume una visión estereotipada de la realidad. No obstante, hay situaciones que nos es muy difícil evitar este tipo de categorización, especialmente cuando tenemos que hablar de tendencias, como es en este caso.

Salvando de antemano esta cuestión, lo cierto es que podemos constatar en diversas investigaciones que las mujeres tienden a ejercer el liderazgo de una forma diferente a la mayoría de los hombres. Esto es lo que lleva a afirmar a numerosos autores y autoras (Apellániz, 1997; Coronel y otros, 1999; Santos Guerra, 2000, etc.), que la manera de pensar, sentir y actuar de las mujeres permite formular la tesis de que las mujeres tienen un estilo de dirección diferente al de los hombres.

Hablan de que la mujer es más consensuadora, más mediadora a la hora de ejercer el liderazgo. Lo curioso en algunas investigaciones es que les surge inmediatamente el "pero". Es decir, que afirman este rasgo como algo positivo, pero a continuación no dejan de aclarar que también esto puede deberse a falta de seguridad personal e independencia de las mujeres. En ningún caso, pensamos, hubiera surgido esta aclaración o matización, si se hubiera tratado de analizar el liderazgo masculino, al que tradicionalmente se le atribuyen cualidades entre las que no estaría la falta de seguridad e independencia. Pero tratándose de mujeres parece necesario incluso desvalorizar o relativizar incluso las apreciaciones positivas. Es una forma soterrada de mantener los privilegios masculinos.

Pensemos que tradicionalmente se consideró que las mujeres no debían ejercer el liderazgo en las organizaciones porque se creía que poseían una serie de rasgos que resultaban contraproducentes para el ejercicio de esas funciones y que según Helgsen (1995) se resumen en los siguientes:

?? Demasiado centradas en dar importancia a los vínculos afectivos

?? Dificultad para considerar el mundo de la dirección como un "gran juego"

?? Escasa capacidad para captar lo esencial del trabajo en equipo, dado su poca afición a los deportes competitivos de equipos como el fútbol

?? Deberian desarrollar un mayor respeto hacia las estructuras jerárquicas y disminuir sus dudas sobre su efectividad 
Sí que podríamos considerar estas características como "rasgos" distintivos del liderazgo femenino en general. Pero lo que habría que cuestionar es si estas características no son las adecuadas para liderar y dirigir las organizaciones actuales.

Al Khalifa (1989) afirma que la teoría "gerencial" aplicada a los centros educativos ha incrementado la posibilidad de asociar conceptos como liderazgo administrativo y masculinidad y, por supuesto, liderazgo y jerarquía. El modelo de "management" que se introdujo en los centros escolares procedente del mundo de la empresa en el Reino Unido, redujo ostensiblemente el número de mujeres que ocupaban cargos directivos en los centros educativos. Y este es el modelo por el que, desgraciadamente, ha apostado la actual administración educativa en su ley de calidad.

Aunque Kaufmann $(1996,183)$ considera que "en la actualidad, las mujeres están en condiciones de revolucionar el lugar de trabajo, precisamente no por dejar a un lado sus valores tradicionales, sino precisamente por expresarlos, en su cotidianeidad laboral", parece que en educación corren malos tiempos para el liderazgo femenino. La nueva ley de calidad de la educación vuelve a una orientación de tipo gerencial y jerárquica en sentido completamente opuesto.

Grimwood y Popplestone (1993) definen el estilo de dirección de las mujeres como abierto, no competitivo, innovador, con un firme sentido de la calidad, centrado en la persona, flexible, comunicativo y persuasivo. Ya en los años 80 Loden (1987) identificaba ocho áreas donde se podían observar las diferencias entre el estilo de gestión masculina y femenina: a) Uso del poder: tienden más a dar poder a los otros/as que a acumular poder personal; b) resolución de problemas: son multimentales, mezclando adecuadamente intuición y racionalidad; c) habilidades interpersonales: saben escuchar, tienen empatía; d) grupos de trabajo: utilizan habilidades de los miembros del grupo en la dirección; e) dirección participativa: están centradas en el grupo y no sólo organizativamente; f) asunción de riesgos: asumen riesgos para perfeccionar la actividad; g) atención a la diversidad: su primera consideración es el personal; h) resolución de conflictos: buscan soluciones que permitan ganar a todos; i) desarrollo profesional: participan en un trabajo aunque no sea parte de su función, pues están implicadas en la búsqueda del desarrollo grupal.

En definitiva, los rasgos más destacados que podríamos señalar y que hacen diferente el uso del poder por parte de las mujeres serían los siguientes:

1. Un estilo más democrático, dialogante, pedagógico, consensuador y mediador. Una actitud más receptiva y participativa.

2. Un liderazgo más multidireccional y multidimensional, favoreciendo el desarrollo de valores y acciones colectivas.

3. Un ejercicio del liderazgo más firme y constante.

4. Más creativas en las propuestas para ejercer la dirección.

5. Más comunicativas y abiertas a las relaciones personales, a las propuestas de sus compañeras y compañeros, a escuchar sin enjuiciar de una forma más comprensiva.

6. Desarrollan políticas de cooperación y participación.

7. Potencian relaciones interpersonales.

8. Disponibilidad para el cambio. 
9. Crean un clima de colegialidad y gestión colaborativa con el profesorado.

10. Construyen redes informales, propiciando la cohesión y los vínculos entre los miembros, alentando a otros a compartir los recursos.

11. Les preocupa el abuso del poder y utilizan la coacción como último recurso.

12. Potencian el "aprender con los demás".

13. Clara preferencia por enfoques consultivos y cooperativos.

14. Habilidad para combinar y reconciliar expectativas y experiencias contradictorias, promoviendo el compromiso colectivo de los miembros de la organización.

15. Desarrollo de políticas de cuidado y apoyo mutuo.

16. Mayor atención a los sentimientos y al uso de una "inteligencia emocional" más sensible a las emociones y situaciones personales de los demás.

En síntesis, dice Kaufmann (1996), las mujeres ponen la parte emocional también en el trabajo. En general las mujeres se hallan más capacitadas para tener en cuenta el lado "humano" de las personas desarrollando para ellos sus dotes intuitivas naturales.

"En las investigaciones revisadas comprobamos cómo el trabajo relacionado con la gestión y el liderazgo desarrollado por las mujeres se caracterizaba por: a) énfasis en las personas y los procesos; b) liderazgo como responsabilidad de todos; c) constitución de estructuras menos burocratizadas, priorizando sobre lo burocrático las relaciones sociales y el sentido de comunidad; d) capacidad para activar conexiones con las personas y el "aprender con los demás"; e) "dejar hacer" potenciando profesionalmente a los colectivos a través de sus propios conocimientos e ideas; f) participación y diálogo como procesos educativos; g) clara preferencia por un enfoques cooperativos y consultivos; h) estilo colaborativo, compartido y no competitivo; i) énfasis en los procesos democráticos; j) desarrollo de políticas del cuidado y apoyo mutuo" (Coronel, Moreno y Padilla, 1999).

Por eso el estilo de dirección de las mujeres -según el estudio hecho por Helgsen (1995)- tiende a ser coherente con estos rasgos de liderazgo femenino. Suelen trabajar a un ritmo más apacible, con pequeñas pausas planificadas durante el día, evitando la acumulación de estrés. No consideran las tareas no planificadas como interrupciones. Tratan habitualmente de ser accesibles, particularmente para los miembros de la organización que trabajan con ellas o a sus órdenes. Poseen una actitud de "cuidado", de "ayudar", de "comprometerse" con lo que hacen. Para ellas lo principal en la organización es "mantener las buenas relaciones", crear un clima de relaciones agradables, donde predomine el apoyo mutuo. Tienen muy en cuenta cuidar las palabras, tener buenas habilidades de comunicación para no herir a los que les rodean, a los compañeros y compañeras de trabajo y, especialmente, a quienes están a sus órdenes. Suelen ser directivas de pequeñas organizaciones, más que de grandes corporaciones. Tratan de estructurar sus organizaciones en forma de "redes" en lugar de una estructura jerárquica, fluyendo así la información en múltiples direcciones. Procuran sacar tiempo de donde sea, para realizar tareas que no estén directamente vinculadas con su trabajo. Raramente anteponen su tarea a sus hijos e hijas, y tampoco pretenden que lo hagan los demás trabajadores o miembros de la organización. Parece que tienen tiempo para leer libros, 
reflexionar y pensar sobre su trabajo de forma intensa. A diferencia de los hombres, dice esta autora, que parecen agotarse en el día a día, las mujeres son más constantes no perdiendo de vista la perspectiva a largo plazo. Si los hombres ven en el trabajo la función primordial de su vida, la mujer, en cambio, considera su función tan sólo como un elemento más de lo que son. Por ello las mujeres estructuran su día tratando de compartir las cosas al máximo, en un proceso cotidiano deliberado.

Si son estas las características que tienden a definir el ejercicio de las mujeres cuando tienen poder, tendríamos entonces que preguntarnos qué pasaría si los cargos de influencia y poder en el mundo estuvieran en manos de las mujeres. Algunas mujeres afirman que la revolución pendiente es la revolución de las mujeres. $Y$ que si los valores asociados tradicionalmente a las mujeres tuvieran mayor reconocimiento social dejarían de existir guerras, industria armamentística y la cultura machista que domina y conforma el ejercicio del poder habitualmente.

Frente a esto, algunos autores afirman que los ejemplos de mujeres ejerciendo cargos de poder o de influencia en la sociedad contemporánea no han sido precisamente ejemplos de este tipo de ejercicio del poder, sino que han sido más duras y más implacables que los hombres. $Y$ ahí está el ejemplo de Margaret Thacher para no ir más lejos, nos dicen.

Sin embargo hay que entender que en la sociedad actual, tal como está organizada, las pocas mujeres que acceden al poder lo tienen que hacer demostrando que son más "duras" que los propios hombres. Para llegar a esos cargos de influencia han tenido que socializarse y mimetizarse en una cultura machista, empleando los mismos métodos y aún más duros que los que han empleado sus competidores hombres. No hubieran sido admitidas en caso contrario.

"La mujer muchas veces asume modelos o patrones masculinos que la sociedad le ha hecho ver como los adecuados para conseguir el éxito. Una mujer firme, franca y directa se la considera agresiva o con demasiadas aspiraciones, lo cual no se aplica a los hombres que muestran el mismo comportamiento. Por todo ello vemos que las mujeres, cuando ejercen cargos de responsabilidad, tienen no sólo que distanciarse de las otras mujeres, sino que tienen que aislar su yo emocional de los hombres" (Coronel Llamas, 1996).

También vemos que cuando una mujer tiene un cierto cargo de poder, las críticas que sufren no suelen ser argumentadas, sino que son utilizadas para descalificarlas dirigiéndose más al yo emocional que al racional ("está histérica", "es que tiene la regla", etc.). Son críticas dirigidas más a lo personal, que a su labor de gestión. Esto se puede ver también cuando en una organización dos mujeres, con cargos de poder, compiten por el liderazgo. Se tiende a interpretar como una cuestión de celos. Mientras que en el caso de los hombres se considera una lucha por el poder.

No obstante, desde la legislación y la lucha de muchas mujeres se está abriendo una brecha de posible normalidad futura en la visión de las mujeres ejerciendo el poder en nuestra sociedad. $\mathrm{Y}$ esto permitirá que esas mujeres cambien la forma y el estilo de ejercer el poder en las organizaciones de las que formen parte.

"En las organizaciones modernas, y con entornos turbulentos y cambiantes, como los que predominan en la mayoría de las sociedades avanzadas, una estructura menos formal y más flexible resulta indispensable. Se sustituiría la ideología militar subyacente por una ideología 'ecológica', enfatizando la importancia de la interpelación entre las cosas y personas. Por todo ello podemos 
concluir que las mujeres pueden llegar a ser mejores directivas que los hombres por su experiencia de implicación activa en la esfera doméstica, que les proporciona una amplia experiencia en la gestión de conflictos, enseñar, guiar, impartir información, gestionar demandas contradictorias, etc." (Kaufmann, 1996, 186).

Pero, para que las mujeres puedan ejercer este liderazgo alternativo, aún hay un escollo previo que solucionar: el acceso de las mismas a esos puestos de poder. Si el liderazgo femenino cumple las expectativas de lo que debería ser un liderazgo positivo, tal como afirman los expertos, ¿por qué los cargos relevantes son desempeñados mayoritariamente por hombres?. Tal como demuestran las estadísticas, en una sociedad, donde la igualdad de oportunidades entre mujeres y hombres parece conseguida legalmente en la realidad cotidiana de nuestras organizaciones, parece una contradicción que no haya paridad en el ejercicio de la dirección en todas las organizaciones e instituciones. Esto es especialmente significativo en las organizaciones educativas, donde las mujeres son mayoría entre los profesionales que ejercen su trabajo en las mismas y sin embargo, la proporción de las mismas en cargos directivos es minoritaria.

Por todo ello nos tenemos que hacer una pregunta previa a la posibilidad de este tipo de ejercicio de la dirección y el liderazgo de forma alternativa o diferente: ¿por qué hay tan pocas mujeres en cargos de responsabilidad y de dirección? $\mathrm{O}$, en otras palabras, ¿qué es lo que dificulta a las mujeres el acceso a desempeñar puestos de dirección o a liderar organizaciones educativas?

\section{EL ACCESO DE LAS MUJERES A LA DIRECCIÓN}

Nosotras nos hemos centrado en analizar el acceso de las mujeres a puestos de dirección en las organizaciones educativas puesto que es el ámbito en el que trabajamos y uno de los contextos organizativos que consideramos más relevantes para estudiar este fenómeno. Pensemos que la profesión educativa tiene un alto porcentaje de mujeres ejerciéndola respecto al número de hombres que trabajan en este campo, especialmente en las etapas de infantil y primaria. $Y$ frente a ello, los datos que nos aportan las estadísticas oficiales se muestran persistentes año a año: el número de mujeres en cargos directivos en los centros educativos es sensiblemente inferior al que le correspondería en proporción a su presencia en esta profesión.

En Castilla y León ${ }^{1}$ hay un total de 18.746 profesores y profesoras que ejercen la docencia en educación infantil, primaria y educación especial en toda la Comunidad, de los que 12.853 son mujeres. En León, la provincia donde hemos centrado nuestras investigaciones sobre este tema, el número total de profesores y profesoras que ejercen en las mismas etapas educativas asciende a 3.470 , de los que 2.470 son mujeres. El porcentaje de hombres directores en la Provincia de León ${ }^{2}$ es de 60,91 \% y de 39,08\% el de mujeres directoras. Aunque este porcentaje es diferente en función del nivel educativo que analicemos: a mayor nivel educativo menor proporción de presencia femenina hay en puestos de dirección.

No sólo es más frecuente encontrar hombres en las tareas de dirección en los niveles superiores del sistema educativo (secundaria frente a primaria o infantil), sino que también nos los encontramos con más frecuencia en los centros más grandes y con mayor número de alumnado y profesorado. De hecho el mayor número de directoras en la provincia de León se concentraba en centros de infantil y primaria con 
dimensiones bastante reducidas (sólo encontramos una directora de un centro con 615 alumnos/as y otra con un centro de 327).

Estos datos parecen ser similares en todo el territorio del Estado. En la investigación realizada por Gairín y Villa $(1999,62-63)$ sobre los equipos directivos de los centros docentes ${ }^{3}$, el cargo de director está ocupado en más de la mitad de las ocasiones por hombres (58\%) y en un menor porcentaje por mujeres (42\%). Cuando este análisis se realiza en función del tipo de centro y del nivel de enseñanza, aparecen algunas diferencias en cuanto al género de la persona que ocupa el cargo de director según el nivel de enseñanza: En la pública predomina el porcentaje de mujeres en los cargos directivos en primaria $(61,5 \%)$ mientras que se invierte drásticamente en los niveles de secundaria (sólo el 33,3\%). Esta misma proporción, u otra muy similar, se mantiene en los demás miembros del equipo directivo en la escuela pública. En la escuela privada el porcentaje de mujeres en los cargos directivos en primaria es menor que en la pública (sólo un $46,2 \%$ ), y en los colegios de secundaria el predominio de los hombres en el cargo de director es abrumador (72,7\%). Ya en los años 80 lo denunciaba Ball (1989) cuando constataba que las mujeres son seriamente desfavorecidas en lo relativo a su carrera por el dominio masculino en las escuelas, aunque la mayoría de los profesores lo niegan con vehemencia.

El tamaño de los centros parece estar en relación con la imagen social de poder que se tiene de ellos, con la dificultad o envergadura de la tarea a desempeñar y con el nivel de lucha y competencia por el acceso y el ejercicio del poder en los mismos. En este sentido, podemos constatar cómo no sólo en los puestos directivos de grandes centros $\mathrm{y}$, especialmente, en los de secundaria no hay mujeres desempeñándolos, sino que los puestos ejecutivos de la administración educativa siguen bajo el dominio de los hombres (inspección, administración central, etc).

Parece pues que las mujeres ocupan más el puesto de dirección en los centros de primaria e infantil de dimensiones más reducidas. Algunos autores (Coronel, Moreno y Padilla, 1999) lo explican por el descenso de prestigio del cargo de dirección en educación. Dado que los hombres tienden a competir por las profesiones más prestigiosas y lucrativas, cada vez van dejando más espacios "desechables" a las mujeres (en este caso, en centros educativos de primaria e infantil). Es la llamada "política de tierra quemada" (las mujeres van tomando lo que los hombres desechan).

No obstante, como estos mismos autores dejan notar citando a Prichard y Deem (1999), hay que ser críticos con el incremento de la participación de las mujeres en los puestos de gestión intermedios de la educación, en parte por considerar que las razones no son otras que su mejor adaptabilidad a los nuevos requerimientos que buscan una gestión más flexible y menos burocrática como requisito para la reestructuración de los servicios públicos. De ahí que la feminización no deba dejar de comprenderse como un efecto de esa presión que el nuevo modelo de gestión (descentralizado, competitivo y flexible) viene a imponer.

A pesar de estas constataciones, lo cierto es que el número de mujeres en puestos de dirección en centros escolares es mucho menor que el que les correspondería en proporción a su presencia. Hay diversas razones para ello que podemos argumentar desde la investigación.

La primera razón histórica, social y cultural que podemos dar de esta menor proporción de mujeres que acceden a cargos directivos no tiene que ver únicamente con el mundo de la educación, sino que es una constante social en todos los campos profesionales actuales: la costumbre social que se convierte en 
norma a la que todos/as nos sentimos inclinados a amoldarnos para no destacar ni hacernos notar. La función de la mujer ha estado reducida de forma tradicional al ámbito de lo domestico y privado, y por ello cuando accede al mundo laboral y al mundo público se encuentra con dificultades no sólo a nivel personal, sino por parte del entorno más próximo (familiar) y del entorno sociocultural más amplio (las organizaciones).

Según Nicolson (1977), para la mujer que logra el acceso a cargos de responsabilidad probablemente aumentarán los problemas y tensiones en su vida. Y no sólo debido a la presión que supondrán las opiniones de su entorno más próximo (pareja, familia...), sino también por la influencia de las redes de apoyo con las que puede contar tanto a nivel práctico como a nivel psicológico (otras mujeres, amistades, etc.). La concepción tradicional mayoritaria en estos dos ámbitos tenderá a orientarla hacia la reducción de sus ocupaciones al ámbito privado.

Además y de forma muy importante están las influencias ideológicas, que provocan unas consecuencias psicológicas en la mujer de carácter negativo, en función de lo que la sociedad espera de ellas como madres de familia, esposas...., lo cual tiende a generar angustia, ansiedad por tener sentimientos de abandono hacia la educación de sus hijos/as; ya que el papel adjudicado a la mujer ha sido el de madre que no puede ser suplantado por otra figura de apego.

Pensemos que, a nivel social, persiste una crítica bastante extendida, o al menos una presión solapada, hacia las mujeres que anteponen el éxito profesional al papel preponderante que se le ha asignado histórica y socialmente: la maternidad. Así la sociedad plantea y define que masculinidad es equivalente a independencia y autonomía. Feminidad significa colaboración, actitud maternal y dependencia.

Un ejemplo de esto es el hecho de que es menos probable que las mujeres en puestos de dirección tengan hijos que los hombres en esas mismas posiciones. Porque mientras que para la empresa un hombre con matrimonio y familia se considera como algo positivo que asegura la estabilidad, una mujer que tiene o puede tener hijos/as son una posible carga -permisos por embarazo, horas de dedicación al trabajo, prioridad en las decisiones, etc.-, lo cual acaba siendo asumido por las propias mujeres y vivido como hándicap en su carrera profesional.

En la investigación que hemos realizado durante el año 2001 con 33 directoras, 98 directores y 291 profesoras de centros educativos de la provincia de León, una de las razones más aducidas inicialmente para no acceder al puesto de dirección, son las responsabilidades familiares. $Y$ es significativo que, de todos los hombres y mujeres que han participado en la investigación, sólo han sido mujeres las que han manifestado expresamente no tener cargas familiares. Alguna de ellas afirmaba que es "una ventaja el no tener hijos/as o responsabilidades familiares que te aten para poder desempeñar la dirección en el caso de las mujeres", mientras que en el caso de los hombres no lo consideraban algo condicionante.

Ligada esta variable a la de su estado civil, hemos podido constatar que si sumamos los porcentajes de las mujeres que ocupan el cargo de directora que están solteras y que no tienen hijos, tenemos que en torno al $18,18 \%$ de las mujeres directivas están en una situación con menos cargas familiares que sus compañeros directores (sólo el 4,6\%). Esto parece indicar que hay un consenso social implícito que supone que las mujeres que no tienen cargas familiares disponen de mayores posibilidades para acceder y desarrollar la labor de dirección. 
"Las mujeres educadas en anteponer sus necesidades a las de los demás sienten más culpabilidad y ansiedad por defender sus propios intereses y necesidades profesionales. No sólo han de demostrar su profesionalidad, sino que además han de defenderse de los intentos de otras personas de excluirlas y dejarlas aisladas; así como de los juicios de valor que los demás establecen sobre sus prioridades. Por tanto, no es solo lo que han tenido que hacer a nivel profesional para demostrar su capacidad sino a lo que han tenido que enfrentarse para conseguirlo, los obstáculos encontrados en otros frentes" (Coronel Llamas, 1996).

Pero es curioso que, en esta investigación, los hombres digan que la dedicación a las tareas directivas les afecta mucho más a sus responsabilidades familiares, refiriéndose a que les quita tiempo, que a las mujeres. Esto parece contradictorio con la mayoría de las investigaciones y discursos teóricos que se mantienen respecto a este tema. Pero, si consideramos que las mujeres tienen que compatibilizar la doble tarea de profesional de la educación y la de cuidadora del hogar y de la familia, cuando se presentan y acceden al cargo directivo se plantean ya seguir manteniendo esa compatibilidad, puesto que no pueden renunciar a ella. Parece, por consiguiente, que los hombres se sienten más culpables en este sentido, ya que constatan que el cargo de dirección les lleva tiempo que restan a la tarea del cuidado del hogar y de la familia, "descargándola" en sus compañeras. También se podría valorar que "justifican" su menor atención a las responsabilidades familiares y al tiempo personal fuera del centro, en función de las exigencias de su cargo directivo.

Una segunda razón estaría relacionada con el hecho de que el concepto de dirección y liderazgo tradicionalmente ha estado ligado a un enfoque jerárquico e individualista. Esta visión tiene como referencia y modelo en la mayoría de los casos la organización industrial. Es un estilo que evalúa el funcionamiento del centro sólo en relación con los resultados obtenidos. En el que la especialización y la división de funciones de los miembros. El/la directivo/a se convierte en un gestor de la organización. Este es un modelo esencialmente masculino. De hecho, los rasgos que se han venido definiendo como propios de este tipo de dirección y liderazgo siempre han estado asociados a las características que tradicionalmente se han atribuido a los hombres (determinación, fuerza, seguridad en sí mismo, etc.). Como dice Santos Guerra (2000) la función de la dirección en los centros educativos se ha identificado con la de quien tiene que garantizar el orden, y así la concepción de autoridad tiene que ver más con el poder que con la educación. "La visión de la dirección que se sustenta en la imposición, en la fuerza, en el dominio; esa cultura atribuye esas características a los varones" (61).

El problema es que esto que, en principio, parecería algo que deberíamos desterrar de nuestras organizaciones, tal como plantea la investigación actual, tendiendo hacia los modelos de liderazgo compartido, sigue presente en el funcionamiento cotidiano de nuestros centros, como se constata en la investigación realizada por nosotras en la provincia de León. Por eso creemos que el cargo de dirección en los centros educativos sigue siendo un cargo poco apetecible para las mujeres en general y para aquellos hombres que se planteen un tipo de liderazgo compartido.

Como hemos visto el estilo de liderazgo femenino es un estilo colaborativo, abierto, democrático, consensuador, de trabajo en equipo y de compartir responsabilidades y trabajo. La mujer no se identifica, por tanto, con la palabra líder. La mujer no busca tanto el éxito social que parece proporcionar el poder o liderazgo. Sí sería más fácil que se planteara acceder a un cargo de dirección cuyo desempeño conllevara fundamentalmente una dinámica de diálogo, de negociación, de trabajo en equipo, de cuidado de las 
relaciones, de desarrollo la inteligencia emocional, etc. Por eso no es deseable para las mujeres acceder a un cargo que habitualmente lo que supone es asumir un rol de autoridad jerárquica (más en sintonía con el estilo masculino), que tiende adeteriorar el clima de entendimiento con sus compañeros y compañeras profesionales, y que muchas veces llega a crear distancia y enfrentamiento afectivo.

Este hecho se puede ver en el porcentaje de nombramientos de directores y directoras "a dedo" por parte de la Administración educativa ${ }^{4}$ : en los centros públicos sólo el $60 \%$ de los directores de colegios de primaria y el $59 \%$ de los directores de institutos han accedido al puesto por elección del Consejo Escolar. El $40 \%$ y el $41 \%$ restante lo han hecho por designación de la Administración educativa. En el caso de la provincia de León, este porcentaje aumenta en el caso de las directoras: un 45,45\% son nombradas por la Dirección Provincial de Educación.

Lo que sí nos pareció muy relevante es que en el caso de las mujeres había un porcentaje mucho más bajo que tomaban la decisión de presentarse por ellas mismas $(21,21 \%$ frente a un $41,53 \%$ de hombres, es decir, casi la mitad). En la mayoría de los casos habían sido animadas reiteradamente por compañeras y compañeros de profesión para que se presentasen. Esto nos llevó a plantearnos si esta no sería una tercera razón de la menor proporción de mujeres en cargos directivos en el sector profesional de la educación. El hecho de no querer acceder a estos puestos de dirección como un mérito personal en el currículum, como una conquista individual del poder.

Es esta una de las diferencias básicas en el acceso de las mujeres a los puestos de dirección en las organizaciones educativas. Tienden a planteárselo como un servicio a la comunidad educativa, como una aportación personal en el intento de mejorar la organización. Esta concepción de su vida laboral, supone una visión de querer estar, no en la cumbre, sino en "el centro de la organización". Sin embargo, los hombres pretenden básicamente acceder a la cumbre de la organización, tener un cargo de poder y de representación institucional.

Esta actitud se halla vinculada con el desarrollo de una intensa racionalidad basada en la competitividad, aprendida desde edades tempranas en los hombres. A los niños (varones) se les suele enseñar, durante su proceso de socialización que lo más importante es ganar, como valor primordial que se halla por encima de la valoración de los vínculos personales o el propio crecimiento. El énfasis está centrado en el "logro profesional", lo cual implica que el tiempo que comparten con sus familias es bastante limitado y sus casas se convierten en "sucursales" de sus despachos, en donde continúan el trabajo que no les ha dado tiempo a terminar en su despacho.

En la investigación realizada hemos descubierto que son los hombres los que mayoritariamente presentan candidatura para ser directores en los centros. Son ellos los que muestran más interés por acceder a ese cargo. Parece pues claro que la lucha por el poder es más manifiesta y explícita en los hombres. Mayoritariamente, cuando la mujer se presenta al cargo de dirección no hay ningún otro candidato, frente a los hombres que en el $81,49 \%$ de los casos se han presentado frente a otros candidatos u oponentes. Además, en esta investigación hemos comprobado que las mujeres tienen menos en cuenta, a la hora de acceder a la función directiva, la remuneración que conlleva o la liberación de la docencia que lleva aparejada, mientras que toman más en cuenta la satisfacción personal y la experiencia adquirida que puede suponer la dirección. 
Una cuarta razón tiene que ver con las expectativas que tenemos sobre cómo van a ejercer el cargo de dirección los hombres y las mujeres, dando por sentado que los hombres se ajustarán más al modelo de dirección que esperamos de ellos. Se da por supuesto que las mujeres lo haríamos peor que los hombres, pues ellos están más acostumbrados y entrenados tradicionalmente a ejercer el poder. Lo hemos constatado en los resultados de esta investigación realizada en León, en los que un $21 \%$ de las profesoras encuestadas afirma explícitamente que, en igualdad de condiciones profesionales, la mujer es discriminada para acceder a un puesto de dirección porque "se presupone la valía superior del hombre".

Por una parte se considera que los hombres tienen ventajas para acceder y desempeñar un cargo directivo y, por otra, se afirma que las mujeres no tienen ninguna. Las tres ventajas que se citan textualmente de los hombres son: "mayor credibilidad por parte del profesorado y alumnado a los directores hombres"; "mayor respeto e incluso más miedo al enfrentamiento directo con los hombres, por lo que el profesorado se vuelve más cumplidor"; y "parece que es más capaz de imponer orden, a los ojos de los alumnos/as e incluso a veces de profesores/as". También afirman un $14 \%$ de las profesoras que otros motivos de la discriminación por razón de género para acceder a un puesto de dirección por parte de las mujeres es que "hay una mayor confianza en la capacidad de toma de decisiones de los hombres", que "se considera que las mujeres tienen inseguridad y miedo para asumir responsabilidades" y "que se acepta más una orden o una corrección si viene de un director hombre" ${ }^{2}$.

"Cuando un hombre realiza mal la tarea de la dirección se suele argüir que es incompetente, que es poco responsable o que tiene problemas que le impiden hacer un buen trabajo. Cuando se trata de una directora, la causa de su mal planteamiento radica en su condición de mujer. (...) Se parte de una expectativa menor por parte de la familia y del entorno sobre la mujer, de una presión diferente sobre ella y de unas valoraciones distintas en el caso de que pretenda alcanzar algo tan legítimo y lógico para sus compañeros varones" (Santos Guerra, 2000, 62).

Por lo que podemos constatar que se tienden a mantener los estereotipos y los prejuicios arraigados en el inconsciente colectivo sobre los comportamientos de ambos sexos en situaciones de poder: "se aplican a la mujer algunos estereotipos especialmente negativos para el ejercicio de la dirección. Las mujeres son consideras histéricas, indiscretas, asustadizas, frágiles..." (Santos Guerra, 2000, 62). Se mantienen estereotipos sobre la mujer y sobre las mujeres: no pueden trabajar juntas, no confían unas en otras, se "despellejan", etc. Pero como dice Nicolson $(1997,196)$ "es la perspectiva masculina la que cree que las mujeres no pueden trabajar juntas". Esto hace referencia a la discriminación interna, la discriminación cultural, los límites que nos autoimponemos desde una tradición cultural que nos marca y nos limita.

Una quinta razón o motivo es la falta de modelos de identificación de mujeres en el poder que lo ejerzan œn características diferentes al de los hombres y que sean referentes para otras mujeres. $\mathrm{Y}$, como plantea Santos Guerra, "los procesos de socialización constituyen un condicionante muy potente en una institución como la escolar, tan asentada en patrones de comportamiento culturales. La mujer ha estado tradicionalmente excluida de puestos de poder" $(2000,61)$.

Durante la investigación realizada, cuando se hablaba en los grupos de discusión de dirección en general y se les pedir a los participantes que describieran la persona en la que estaban pensando, coincidían en un amplio porcentaje en identificar un hombre, asumiendo la imagen de dirección con modelos 
masculinos. Como hemos visto, los "rasgos" y características que se asocian a los líderes, son más acordes con los que tradicionalmente se han atribuido a los hombres.

Además, esta falta de modelos de identificación de las mujeres se puede ver también en el hecho de que una gran mayoría de los directores habían sido anteriormente jefes de estudio (67\%), frente a las directoras de las cuales sólo un 36\% había sido jefa de estudios antes de ser directora. Lo cual parece indicar que es importante el entrenamiento para poder desempeñar un puesto directivo. En la medida que se forma parte de un equipo de dirección y se van conociendo sus funciones y se va quitando el cierto recelo o miedo a su ejercicio, será más fácil volver a participar en un cargo directivo. Esto es importante para las mujeres, dado que si no se entrenan en la participación en estos cargos, se convierte en mucho más difícil el acceso a los mismos. Esta es una de las barreras que se constataba en este estudio. El entrenamiento en tomar decisiones, en organizar, hablar en público, el entrenamiento en decidir, etc., son aprendizajes fundamentales para ejercer los puestos directivos, en los que tradicionalmente se les ha formado mucho menos a las mujeres.

El cargo del equipo directivo que la mujer más habitualmente ha desempeñado con anterioridad es el de secretaria (42\%) frente a un $36 \%$ de directores que habían sido secretarios. Parece que se mantiene esa correlación tradicional de "subordinación" de las mujeres en el campo organizativo, ligado a cargos de secretaria más que al ejercicio de la jefatura de estudios.

Hay una directiva europea para promover la igualdad, que establece que, en el acceso a cargos de responsabilidad en aquellos sectores en que la mujer está subrepresentada, a igual formación se priorice a las mujeres. Esto facilitará que tengan este entrenamiento que es esencial.

Pero esta falta de modelos de identificación, se constata en el propio uso tradicional del lenguaje. De hecho el propio lenguaje niega esa posibilidad de identificación al no poder poner en femenino determinados cargos. Es sorprendente que en la profesión educativa, donde el modelo imperante debería ir por delante en cuanto a ruptura de diferencias o connotaciones discriminatorias por género, se siguen utilizando términos masculinos para designar cargos aún cuando estén desempeñados por mujeres: jefe de departamento, jefe de estudios, etc.

Pero esto es algo que viene pasando en la mayoría de las investigaciones y estudios genéricos (que no aborden específicamente el tema de género) sobre la dirección y el liderazgo. La utilización del lenguaje siempre alude implícita y explícitamente a los hombres. Igualmente los tratados teóricos sobre dirección y liderazgo están escritos genéricamente en masculino, y el contexto que progresivamente se va generando en su lectura induce a pensar en hombres como personajes que encarnarán esas funciones o asumirán ese papel. Así, los primeros estudios sobre el liderazgo trataron de estudiar la personalidad del líder a través del análisis de personajes históricos que se habían revelado como líderes, tratando de descubrir aquellas características que poseerían únicamente ciertas personas y que las convierte en líderes. Esta tendencia fue denominada como "teoría de los grandes hombres". Parece que no era entendible que hubiera "grandes mujeres".

"Las instituciones escolares, aunque mixtas, pueden calificarse de organizaciones androcéntricas. La definición de la escuela refleja los valores y significados de la cultura de los hombres. El lenguaje y la estructura de la educación son modelados, de forma predominante, por el patriarcado" (Santos Guerra, 2000, 56). 
Otro dato fruto de esta investigación y que podemos considerar también una sexta razón de la situación actual en cuanto a la proporción de mujeres que hay en puestos directivos en las organizaciones educativas hace referencia a la convicción de que actualmente ya no hay discriminación en la sociedad en que vivimos por parte de buena parte de esa sociedad. Aunque un $60 \%$ de las directoras encuestadas y un $52 \%$ de las profesoras sí consideraban que son elegidos mayoritariamente hombres para desempeñar cargos directivos, sólo un 30\% de ambas afirman que hay discriminación por razón de género para acceder a un puesto de dirección. En el caso de los directores el $30 \%$ de ellos afirma que sí se elige mayoritariamente a hombres para los puestos de dirección, pero casi un $60 \%$ dicen que no hay discriminación por razón de género en esta elección. Lo que nos tenemos que preguntar es cómo se puede tener esta convicción cuando los datos, como hemos visto, desmienten esa afirmación. ¿Cómo es posible no ser consciente una realidad tan evidente?

Esta convicción tan arraigada, de que ya todos y todas somos iguales, tiende a explicar la dificultad de acceso de las mujeres a puestos de dirección por problemas de personalidad o de preparación de ellas.

Según Faludi (1992), desde todos los ámbitos, recibimos el mensaje de que somos iguales, de que hemos ganado la batalla de la igualdad. Esto significa que el discurso de la igualdad con el que nos bombardean constantemente ha hecho mella, nos lo hemos creído. A lo largo de nuestra escolaridad, hemos compartido escuelas, nos hemos educado juntos mujeres y hombres. Por lo tanto somos iguales. La legislación también recoge igualdad de derechos para hombres y mujeres. Por lo tanto, si algo falla debe ser por razón de la inadecuación de las mujeres. Ya no sólo es que las mujeres no quieran, sino que no valen para ello (Eguskiza, 1996).

Es el doble discurso presente en el análisis de la realidad. Mientras el discurso dominante formal en las organizaciones está basado en la neutralidad e igualdad, la persistencia de asimetrías en relación al género indica la continuación o incluso el reforzamiento de la desigualdad de género en las organizaciones (Benschop y Doorewaard, 1998), en su funcionamiento cotidiano, en su cultura.

Estamos en una cultura tradicionalmente tan patriarcal que no nos permite pensar una sociedad en condiciones de igualdad. No hay experiencias, ni referentes, ni tradiciones que nos muestren cómo sería una sociedad organizada paritariamente. $Y$ tendemos a negar las realidades que no se ajustan a las concepciones que consideramos que "deben ser". Está claro que acabamos viendo, lo que queremos ver. Los argumentos y razones que aducen tanto directores como directoras confirman que esta tradición patriarcal impide la conciencia sobre estas discriminaciones indirectas, culturales, en las que crecemos y nos movemos hombres y mujeres. Es el peso de la cultura.

Y esto se perpetúa, incluso en los argumentos que se dan por parte de algunas mujeres y bastantes hombres que aluden a que ya hay mujeres en puestos directivos y que si no hay más será porque no quieren. Esto es lo que Santos Guerra (2000) denomina el "mito de la excepción":

“como elemento eliminador de conflicto se presenta el 'mito de la excepción': si algunas han sido y son directoras, ¿por qué no lo han podido ser las demás? Argumento falaz frecuentemente utilizado para negar la discriminación” (55).

Parece pues que no se quiere investigar las causas estructurales. No nos queremos adentrar en los mecanismos implícitos de organización y funcionamiento de las instituciones que generan estructuras injustas de relación y participación. Es más fácil achacar estas situaciones a razones personales, a problemas individuales. Hemos conseguido la igualdad, pero son las mujeres las que no quieren acceder a 
puestos de dirección. Tienen otras prioridades, les importan más otras cosas, el cuidado del hogar, la atención de la familia, etc.

Estas explicaciones son la psicologización individualista de los problemas que van calando en las generaciones jóvenes y que les impiden seguir luchando decididamente para conseguir otra forma de dirigir y liderar las organizaciones. Nos han convencido de que no es posible otra forma de dirigir y lo hemos asumido como propio.

La séptima razón explicativa de esta desigualdad por razón de género en el acceso de las mujeres a los puestos de dirección hace referencia a la baja autoestima que se ha fomentado tradicionalmente en las mujeres ante el ejercicio del poder. Esto tiene que ver con las percepciones que nos hemos ido formando en torno a las tareas directivas y cómo éstas afectan a la hora de decidir participar o no, "colaborar" o no. Y estas percepciones tienen mucho que ver con la idea de poder asociado a ellas y la relación que tradicionalmente las mujeres han ido estableciendo con éste. Esto ha sido especialmente estudiado en las organizaciones empresariales donde la mujer consideraba que iba a ser menos respetada y valorada por sus subordinados que otros hombres en cargos directivos. Esta prevención por la posible respuesta de los miembros de la organización ante el ejercicio del poder por parte de una mujer, ha provocado en muchos casos un "círculo vicioso" en el que la mujer se ha sentido más insegura para ejercer el liderazgo.

Sin embargo, en el terreno educativo, la débil autoestima o la infravaloración de la mujer en el ejercicio de la dirección no parece ser un problema. En la investigación hemos podido constatar cómo hay incluso más directoras (un 27\%) que se consideran a sí mismas como "buenas directoras", frente a un $15 \%$ de directores que se autovaloran como buenos en el ejercicio de esta función. Lo cual supondría que, una vez en el cargo, las mujeres tienden a tener una valoración positiva y una autoestima elevada respecto a su labor profesional, y que es en la consideración previa a su acceso cuando se detectan más problemas de autoestima asociados a prejuicios culturales y sociales muy arraigados en toda la población y, por tanto, también en las propias mujeres.

A pesar de ello, no podemos dejar de reseñar que las mujeres se sienten mucho más criticadas y cuestionadas que los hombres en su tarea de dirección. En este sentido, parece que las mujeres que llegan a cargos de responsabilidad se sienten más observadas, sus fallos se les dan mayor relevancia y estos fallos tienden a achacarse al hecho de ser mujeres o a alguna característica tradicionalmente atribuida a la "condición femenina". De hecho un $18 \%$ de las encuestadas consideraron que las mujeres sufren un mayor nivel exigencia para acceder a cargos directivos. También es importante constatar, en este sentido, que casi una cuarta parte de las directoras constata en su ejercicio profesional que se acepta más una orden o corrección que provenga de un hombre, por lo que el ejercicio de la dirección así se convierte en una constante lucha por demostrar que se tiene autoridad por parte de las mujeres. $\mathrm{Y}$ a las directoras les importa menos reconocer que piden ayuda y asesoramiento en el ejercicio de su cargo directivo que a los directores. Lo curioso es que un $9 \%$ de ellas pide esa ayuda preferentemente a hombres, mientras que los directores en ningún caso afirman hacerlo preferentemente con hombres.

Podemos concluir con una frase de una directora en la investigación: "en general, sólo se acepta y valora el modelo masculino en los puestos directivos, mientras que la forma habitual de las mujeres de ejercer el poder (consenso, diálogo, atención a diferentes puntos de vista) es visto como un indicativo de 
debilidad e inseguridad". Pero los trabajos llevados a cabo desde una perspectiva de género reconocen que las mujeres posiblemente cuenten con habilidades comunicativas y sociales más adecuadas para las exigencias de unas organizaciones participativas y democráticas.

Por eso nos tenemos que preguntar de nuevo si otra educación no sería posible si las mujeres ejercieran el poder en las organizaciones educativas.

Si consideramos que las mujeres tienden a un tipo de liderazgo más consensuador, más de colaboración, en el que se prioriza crear un clima de entendimiento y participación, donde se fomenta la comunicación, el debate, el diálogo y el trabajo en equipo, cómo es posible que haya tan pocas mujeres en puestos directivos por elección de sus compañeros y compañeras. ¿ $O$ es que lo que en el fondo se demanda en los centros educativos es un director que mande, que imponga disciplina y orden con autoridad personal que nos permita permanecer cómodamente apoltronados sin tener que arriesgarnos a implicarnos y tomar decisiones, criticando desde la retaguardia?

Es necesario por ello seguir investigando para ampliar la perspectiva de los estudios centrados en el género. Esto supone, estudiar y comprender el papel de hombres y mujeres dentro de las organizaciones educativas. También es necesario, trabajar con mujeres para conocer qué hacen en el desempeño de cargos directivos, y para conocer su conquista, su adquisición y la generación de poder. No podemos permitir que en la teoría organizativa siga primado una ideología y valores masculinos, en función de los cuales los aspectos relacionados con el género femenino quedan relegados a un segundo plano. Es necesario que el género sea tenido en cuenta a la hora de explicar la estructura organizativa de una entidad.

La perspectiva de género ha puesto en evidencia la necesidad de avanzar hacia una visión de las escuelas en su conjunto, como comunidades democráticas.

El siglo que hemos empezado se abre con nuevas conquistas de igualdad para la mujer. En las décadas precedentes se han reconocido los derechos de las mismas como parte de los derechos humanos, en la medida que se exige la no-discriminación por razones de sexo. El principio de igualdad a nivel legal, ha generado importantes cambios en los derechos de las mujeres y su posibilidad, al menos teórica, de acceder a las diferentes esferas sociales.

Esta igualdad legal no será real sin el apoyo explícito de políticas que desarrollen planes que garanticen o favorezcan el acceso de la mujer a las diferentes áreas de poder en igualdad de oportunidades. Pero las mujeres, como señalan diversas autoras, no ocupamos o participamos del núcleo del poder, ya sea económico o político, y el acceso a los máximos niveles de responsabilidad sigue estando lleno de obstáculos, e incluso vedado para nosotras como hemos visto. No obstante, a la vez que constatamos esta realidad somos conscientes de que la participación en estos núcleos de poder es un requisito imprescindible para la construcción de una democracia más plena y verdadera.

Analizando la información existente sobre el tema y contrastándola con los materiales utilizados en esta investigación hemos llegado a la conclusión de que la mujer en el ámbito de la dirección educativa puede participar activa y críticamente pero siempre con el intenso esfuerzo de superar una serie de barreras sociales y culturales que son las que nosotras creemos le impiden acceder y desarrollar un puesto directivo. Suprimiendo este tipo de barreras lograremos que las organizaciones educativas del futuro tengan como eje 
principal a la persona y estén formadas por seres humanos integrales, siendo esto uno de los principales y más importantes retos tanto de mujeres como de hombres, y en general de nuestra sociedad.

Y en esta concepción de liderazgo sí que parece que las mujeres tienen un papel protagonista no sólo para desempeñarlo de una forma más oportuna, sino también para enseñar a los hombres cómo desarrollar un tipo de liderazgo más colaborador, consensuador, dialogante y participativo. En nuestras manos está el futuro de la educación y no podemos desaprovechar esa oportunidad.

\section{BIBLIOGRAFÍA}

AA.VV. (1998). Principales dificultades de la dirección de centros educativos en los primeros años de ejercicio. Bilbao: ICE de la Universidad de Deusto.

AL KHALIFA, E. (1989). Management by Halves: Women Teachers and School Management. En Lyon, H. y Migniuolo, F.W. (Ed.). Women Teachers. Issues and Experiences. Milton Keynes: Open University Press.

ALVAREZ,M. Y SANTOS, M (1996). Dirección de centros docentes: gestión por proyectos, Madrid: Escuela Española.

ASTIN, H. y LELAND, C. (1991). Women of influence women of vision. San Francisco: Jossey-Bass.

BALL, S.J. (1989). La micropolítica de la escuela. Hacia una teoría de la organización escolar. Madrid: PaidósMEC.

BASS, B.M. (2000). El futuro del liderazgo en las organizaciones que aprenden, En AA.VV. Liderazgo y organizaciones que aprenden (III Congreso Internacional sobre Dirección de Centros Educativos 331361) Bilbao: ICE Universidad de Deusto.

BENSCHOP, Y. y DOOREWAARD, H (1998) Covered by equality: The gender subtext of organizations. Organization Studies, 19 (5) 787-805.

BOLÍVAR, A. (2001). Liderazgo educativo y reestructuración escolar. Ponencia en el I Congreso Nacional sobre Liderazgo en el Sistema Educativo Español (95-130). Córdoba: Grupo de Investigación sobre Liderazgo Educativo.

BRUNNER, C. (2000) Unsettled moments in settled discourse: women superintendents' experiences of inequality. Educational Administration Quarterly, 36 (1) 76-116.

BRUNNER, C. (1998) Sacred dreams: Women and the superintendecy. New York: State Univ. of NY Press.

CARRASCOSA, A. (2002). Leyes masculinas con voz femenina. Equis Equis, 3, 13.

CERRADA, A. y SEGURA ,C (Ed.) (2000). Las mujeres y el poder: representaciones y prácticas de vida. Madrid: al-Mudayna

CORONEL, JM. (1996). La investigación sobre el liderazgo y procesos de cambio en los centros educativos. Huelva: Servicio de Publicaciones de la Universidad, 1996.

CORONEL, J.M., MORENO, E. y PADILLA, Ma.T. (1999). La gestión y el liderazgo como procesos organizativos: contribuciones y retos planteados desde una óptica de género. Revista de Educación

DíEZ GUTIÉRREZ, E.J. (2002). Dos visiones de la educación y la calidad. Cuadernos de Pedagogía, 318, noviembre 2002, 84-86.

DRAKE, P. y OWEN, P. (Eds). (1998). Gender and Management issues in Education: an international perspective. London: Trentham Books 
EGUSKIZA, Ma.J. (1996). Mujeres y Gestión Educativa. En Gairín, J. y Darder, P. (Coords.). Organización y Gestión de Centros Educativos (292/85-292-91). Barcelona: Praxis.

FALUDI, S. (1992). Backlash. The Undeclared War Against Women. Londres: Chatto \& Windus.

FENNELL, H. (1997). A passion for excellence: Feminine faces of leadership. Annual meeting of the AERA, Chicago, IL, .

GAIRÍN, J. Y VILLA, A. (Eds.) (1999): Los equipos directivos de los centros docentes. Análisis de su funcionamiento. Bilbao; Universidad de Deusto.

GAIRIN,J. (1999). Estudios de desarrollo organizativo: de la organización como estructura a la organización que aprende en LORENZO, M. y Otros: Enfoques comparados en organización y dirección de instituciones educativas, Granada, Grupo Editorial Universitario.

GAIRÍN, J. y VILLA, A. (Eds.). (1999). Los equipos directivos de los centros docentes. Análisis de su funcionamiento. Bilbao: Deusto.

GILLIGAN, C. (1982). In a different voice. Psychological theory and women's development. Cambridge: Harvard University Press.

GIMENO SACRISTAN,J. (1995). La dirección de centros. Análisis de tareas. Madrid: CIDE.

GOLDRING, E. y CHEN, M. (1992). The feminization of principaship in Israel: the trade off between political power and cooperative leadership. Washington and London: Falmer Press.

GRAY, H. (1987). Gender considerations in school management: masculine and femenine leadership styles.

School Organization, 7 (3) 297-302.

GRIMWOOD, C. y POPPLESTONE, R. (1993). Women, Management and Care. Londres: McMillan.

GROGAN, M. (1997). Voices of women aspiring to the superintendency. Albany, NY: Suny Press.

HALL, V. (1996). Dancing on the Ceiling: a study of women managers in education London: Paul Chapman.

HELGSEN, S. (1995). The female advantage: Women's ways of leadership Toronto: Doubleday Currency.

HERSEY, S. y BLANCHARD, M. (1993). Management of Organizational Behaviour. Londres: Routledge.

HURTY, K. (1995). Women principals leading with power. New York: SUNY Press.

JACOBS, Jerry. (1999). Gender and the stratification of colleges. The Journal of Higher Education, 70 (2) 161187.

KAUFMANN, A. (1996). Tercer milenio y liderazgo femenino. En NUÑO GÓMEZ, L. (Coord.). (1996) Mujeres: de lo privado a lo público (177-186). Madrid: Técnos.

KATZ, D. y KAHN, R.L. (1978). The social psychology of organizations. New York: Wiley.

LIPS, H. (1991). Women, Men and Power. Mountain View, CA: Mayfield Publisbing Comp.

LODEN, M. (1987). Dirección femenina: cómo triunfar en los negocios sin actuar como un hombre. Barcelona: Hispano Europea.

LÓPEZ YÁÑEZ, J. (1993). Proyecto Docente. Sevilla: Universidad de Sevilla.

LORENZO DELGADO, M. (2001). Visión actual del liderazgo en el sistema educativo español. Ponencia en el

I Congreso Nacional sobre Liderazgo en el Sistema Educativo Español (65-82). Córdoba: Grupo de Investigación sobre Liderazgo Educativo.

LORENZO, M. (1999). El liderazgo educativo en los centros docentes. Madrid: La Muralla.

MEC (1994). Centros Educativos y Calidad de la Enseñanza. Madrid: MEC.

MERTZ, N. (1995). Journey into the theoretical gap: A study of female high shool principals lived experience. Annual meeting AERA, San Fco. 
MUÑOZ-REPISO, M., MURILLO, F.J. y otros. (2001). Aportaciones de las Teorías de la Organización al nuevo movimiento teórico-práctico de Mejora de la Eficacia Escolar. Revista española de Pedagogía, 218, 6984.

MURILLO, F.J. (2000). La investigación sobre eficacia escolar en España. En Villa, A. (Coord.). Liderazgo y organizaciones educativas que aprenden (1007-1020). Bilbao: Mensajero.

NICOLSON, P. (1997). Poder, género y organizaciones. Madrid: Narcea.

NUÑO GÓMEZ, L. (Coord.). (1996). Mujeres: de lo privado a lo público. Madrid: Técnos.

PRICHARD, C. y DEEM, R (1999). Wo-managing further education; gender and the construction of the manager in the corporate colleges of Englad. Gender an Education, 11 (3) 323-342

REYNOLDS, C. (1995). Feminist frameworks for the study of administration and leadership in educational organizations. En Reynolds, C. y Young, B. (Eds) Women and Leadership in Canadian Education (3-17). Calgary: Detselig.

SÁNCHEZ APELLÁNIZ, M. (1997). Mujeres, dirección y cultura organizacional: Madrid: CIS.

SANTOS GUERRA, M.A. (2000). Yo tengo que hacer la cena. La mujer y el gobierno de los centros escolares.

En Santos Guerra, M.A. (Coord.). El harén pedagógico. Perspectiva de género en la organización escolar (53-69). Barcelona: Graó.

TSOLIDIS, G. (1996) Feminist theorisations and difference -a case study related to gender education policy. British Journal of Sociology of Education, 17 (3) 267-277.

WILSON, F. (1996) Research note: Organizational Theory: Blind and Deaf to gender? Organization Studies, 17 (5) $825-842$.

ZALEZNIK, A. (1991). La mística del management. Barcelona: Grijalbo.

1 Datos aportados en el año 2001 por la Consejería de Economía y Hacienda, a través de la Dirección General de Estadística de la Junta de Castilla y León.

2 Datos aportados por la Dirección Provincial de León a fecha de octubre de 2001,

3 Investigación sobre una muestra formada por 52 directores/as, 67 jefes/as de estudio, 46 secretarios/as y 72 otros miembros del equipo directivo.

4 Estudio del INCE del año 2001, Evaluación de la función directiva 2000.

5 Es sorprendente que en ningún momento nadie de las encuestadas considere que se aceptaría más una orden o una corrección si viniera de una directora mujer. 


\title{
Contactar
}

Revista lberoamericana de Educación

\author{
Principal OEI
}

